\title{
Niveles experimentales de conflicto social e influencia minoritaria: Límites en la definición de influencia indirecta
}

\author{
Luis GonzÁlez Fernández, \\ EUGENIO GARRIDO MARTÍN \\ Universidad de Salamanca
}

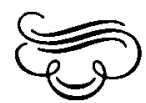

Resumen

El propósito de la presente investigación es desarrollar una jerarquia experimental que estudie las caracteristicas más importantes del conflicto social en los procesos de influencia minoritaria. También se bace un análisis de los límites de la influencia latente minoritaria. Se defiende asi la idea de que la influencia indirecta de las minorias reposa sobre una relación de naturaleza binaria no transitiva entre las posiciones minoritarias y las opiniones y actitudes objeto de influencia. Para ello se elaboró una serie de experimentos que combinan las siguientes variables, pertenencia categorial y presentativi. dad de la fuente minoritaria, en un diseño factorial $3 \times 2$. Los sujetos, 107 escolares (110 mujeres y 87 varones) fueron distribuidos en seis condiciones experimentales resultantes de la combinación de dos clases de variables: fuente de influencia perteneciente a un intragrupo, a un extragrupo, o a una minoria sin categorización social; fuente minoritaria representativa o no representativa. Los resultados subrayan la importancia del conflicto social en la producción de la influencia minoritaria y parecen confirmar la bipótesis de los límites en la influencia indirecta minoritaria.

\section{Abstract}

The purpose of the present research is to develop an experimental bierarchy which studies the most important characteristics to social conflicts in minority influence processes. It is also made an analysis of the boundaries in minority latent influence. With this we uphold the idea that the indirect influence of the minorities rests on a relation of binary no-transitive nature between the minority positions and the opinions or attitudes object of influence. In order to do this, an experiment was elabonated; it combines the following variables, categorial and representative ownership of the minority sounces; in a $3 \times 2$ factorial design. The subjects, 107 bigh schools (110 girls and 87 boys), are distributed in six experimental conditions resulting from crossing two kinds of variables: influence source belonging to the ingroup, to the outgroup or to the minority without social categorization; representative or not representative minority source. The results underline the importance the social conflict in the production of minority influence and seem to confirm the bypothesis of the limits on indirect minority influence.

Dirección del autor: Facultad de Psicología. Avda. de la Merced, s/n. 37005 Salamanca. 


\section{INTRODUCCION}

Las investigaciones sobre los procesos de influencia minoritaria formuladas desde una perspectiva interaccionista, que concibe las normas sociales como relativas y pone el acento en los mecanismos de transformación, de innovación, no tienen más de veinte años (para una revisión ver: Maass y Clark, 1984; Moscovici, Mugny y Van Avermaet, 1985; Moscovici y Mugny, 1987). Un gran número de estas investigaciones se centran en el estudio del conflicto como núcleo explicativo de los procesos de influencia social.

El modelo genético (Moscovici, 1976) sitúa los mecanismos de innovación en el conflicto que las minorías introducen en el sistema social. El instrumento de creación y gestión de este conflicto es el estilo de comportamiento adoptado por la minoría. En otras palabras, una minoría que sostiene sus puntos de vista organizando su comportamiento de manera consistente, diacrónica y sincrónicamente, genera un conflicto por la aparición y defensa de una norma alternativa en el campo social. De la resolución de este conflicto depende la obtención de influencia por parte de las minorías. Así pues, la influencia se explica en última instancia desde los comportamientos ocurridos durante la interacción y no desde algún tipo de dependencia previa a la situación de influencia (Montmollin, 1977).

La noción de conflicto sigue siendo esencial dentro del armazón conceptual del modelo psicosociológico propuesto por Mugny (1981). Para este autor es necesario considerar los procesos de influencia social, y minoritarios en particular, inmersos en el tejido de relaciones sociales intergrupo. Un primer paso en esta constatación lo constituyó el afirmar que al menos tres entidades están implicadas en todo proceso de innovación: Poder, Población y Minoría (Mugny y Papastamou, 1976-77). El poder constituye la entidad dominante en una relación asimétrica de dominio y, a menudo, simbolizado a través de las normas consideradas como mayoritarias en una situación particular. La población está formada por los sujetos blanco de influencia, tanto de las minorías como del poder. Las minorías son los grupos que aspiran a modificar las normas mayoritarias, definidas desde un punto de vista númerico, en relación con la norma que proponen como alternativa. Por lo tanto, la influencia social no se reduce a una oposición binaria de mayoría/minoría. La distinción entre estas tres entidades permite diferenciar los planes de acción que pueden desarrollar los distintos agente sociales: estilo de comportamiento y estilo de negociación. El primero califica las estrategias frente al poder, mientras que el segundo define la estrategia que la fuente minoritaria debe adoptar frente a la población. Esto es posible por la coexistencia de relaciones de diferente naturaleza. El poder mantiene una relación de dominio con la población. Entre poder y minoría se establece una relación de antagonismo; para ser una alternativa en un sistema social la minoría debe bloquear toda negociación del conflicto que genera con el poder. Finalmente, entre la minoría y la población se instaura un proceso de influencia social.

El reconocimiento de la inserción de los procesos de influencia social dentro del tejido de relaciones grupales hace necesario acudir a una noción que explique el mecanismo por el cual interviene este contexto intergrupo. Dicho concepto es el de indentidad psicosocial. De acuerdo con el modelo de relaciones intergrupo, en los procesos de influencia social la fuente es percibida por los sujetos blanco dentro de un campo social categorizado (Doise, 1976; 1982). 
Además, la simple categorización de los individuos como intragrupo o extragrupo, por mínima que sea, es suficiente para implantar un favoritismo intragrupoy una discriminación extragrupo a nivel evaluativo y comportamental. Ambos procesos tendrían por función el asegurar una identidad social positiva a los miembros del grupo cuando en una competición social simbólica se oponen a un extragrupo (Turner, 1981). La influencia social expresaría, de este modo, el grado en el cual los sujetos están dispuestos a identificarse con la fuente; es decir, a reconocerse como próximos a esa categoría y asignarse las características y evaluaciones que le son propias. Aplicado a los fenómenos de influencia minoritaria, permite suponer que el impacto será mayor cuando exista una relación privilegiada entre fuente y blanco, sobre la base de una identidad común, explicado por un proceso de comparación social. Una cierta comunidad de identidad entre blanco y fuente asegura o refuerza una mayor influencia (Mugny y Papastamou, 1982).

Sin embargo, para los autores de la Escuela de Ginebra la explicación de los procesos de influencia minoritaria desde el punto de vista de la comparación social no da cuenta de todo el fenómeno. En la fase inicial del proceso de influencia la minoría aparece como alternativa en el campo social por la creación de un conflicto al plantear la ruptura con la norma dominante. Su identidad social está sujeta a percepciones negativas y será, necesariamente, categorizada como extragrupo, o por lo menos en el límite del intragrupo. Sujetas a este proceso de discriminación e insertas en un conflicto de identificación, el potencial de influencia de las minorías debería reducirse o anularse. No obstante, puede haber un efecto contradictorio, desde la perspectiva del modelo intergrupo, ya que una minoría, que al aparecer como alternativa es forzosamente categorizada como extragrupo, al menos en la dimensión que les diferencia, puede obtener, no obstante, influencia a nivel indirecto. En efecto, un conjunto de investigaciones (Nemeth y Endicott, 1976; Mugny, Pérez, Kaiser y Papastamou, 1984) demuestran que el sesgo del favoritismo intragrupo y la discriminación extragrupo no es sistemático. Además, Moscovici, Mugny y Pérez (1984-85) han comprobado que una minoría categorizada como extragrupo y con su discurso denegado - connotado como irreal, inverosímil- puede obtener influencia.

De este modo, los autores de Ginebra plantean la necesidad de formular un modelo integrativo que articule las explicaciones de los procesos de influencia minoritaria y los procesos de relaciones entre grupos (Mugny y Pérez, 1986). La identidad categorial de la fuente no sería la responsable última de la influencia obtenida, sino que establecería los límites de que dispone la minoría para crear un conflicto de mayor o menor intensidad. Una minoría intragrupo posee un margen de libertad mayor que una minoría extragrupo, que debe ser menos conflictiva. Frente al conflicto inducido por una minoría los sujetos blanco raramente son pasivos, llevan a cabo una reconstrucción del conflicto en una doble dirección, motivacional y sociocognitiva. El origen del conflicto sociocognitivo está en la oposición entre los atributos que se introducen en una situación de influencia y la matriz de identidad previa de los sujetos. La resolución de este conflicto pasa por una readaptación de la matriz de identidad, redefiniendo conjuntamente los atributos y las categorías. El conflicto es, pues, el elemento central en la explicación de los fenómenos de influencia minoritarios, tanto en las dinámicas de identificación asociadas a los procesos de comparación social, en una aproximación de matiz intergrupo que da cuenta de la influencia directa, como de la actividad cognitiva de validación a través de la cual los sujetos blan- 
co de influencia redefinen el campo social, introducen nuevos argumentos, nuevas significaciones y conduce a un impacto de naturaleza indirecta.

Tanto en el modelo genético como en el modelo psicosociológico la relación entre la intensidad del conflicto y el grado de influencia obtenida es considerada como una función líneal ascendente. A mayor intensidad del conflicto mayor impacto social. La evolución de las investigaciones sobre influencia minoritaria conduce a una reformulación de esta relación. En el modelo integrativo se redefine la relación entre conflicto e influencia social como una función cuadrática, en forma de «U» invertida. Un nivel mínimo o máximo de conflicto no produce influencia. En palabras de Mugny y Pérez (1985): «La influencia dependerá de la capacidad de generar un conflicto (por la consistencia) que puede, por otro lado, negociarse al menos parcialmente; en suma de un conflicto óptimo, ni muy fuerte, ni muy débil.» (pág. 3). Esta curva es básicamente heurística, la mayor o menor intensidad debe interpretarse de acuerdo con las diferentes naturalezas del conflicto. Uno de los parámetros pertinentes para entender estos puntos de inflexión son las diversas categorizaciones de la fuente de influencia.

La hipótesis general de este estudio construye una jerarquía experimental de niveles de conflicto, en base a su naturaleza, esperando que la influencia obtenida sea más importante en aquellos grupos en los cuales existe un nivel de conflicto óptimo. Pretendemos interpretar los diferentes puntos de inflexión de la curva resultante entre influencia obtenida e intensidad de conflicto de acuerdo con los distintos significados de la naturaleza del conflicto que se plantea. En un plan factorial se variará la identidad categorial (intragrupo versus extragrupo versus sin categorizar) y el grado de representación de la fuente minoritaria (representativa versus no representativa). Dos muestras de sujetos de identidad diferente permitirán, al mantener la minoría en ambas una posición constante, modular la intensidad del conflicto según se argumente a favor de la identidad de los sujetos o en contra de la misma.

Antes de exponer la hipótesis del presente trabajo conviene subrayar que metodológicamente hay que considerar la distinción entre influencia pública/privada, inmediata/diferida o directa/indirecta, si se quiere estimar el impacto de las minorías. Estos términos han sido utilizados desde las primeras investigaciones sobre influencia minoritaria (Moscovici, Lage y Naffrechoux, 1969) de manera indistinta, como sinónimos. Sin embargo, creemos que, como afirman Mugny y Pérez (1986), el status de estas distinciones metodológicas tiene consecuencias teóricas. La distinción entre influencia pública y privada expresa una cierta opacidad. El carácter público resalta los costes sociales de una eventual identificación, mientras que la respuesta privada escapa, en cierto modo, al control social por parte de la mayoría, quedando más lejana una posible comparación social. La diferencia entre influencia inmediata y diferida supone tomar en consideración los efectos de la variable tiempo. Los sujetos blanco desarrollarían, con el paso del tiempo, una actividad cognitiva de validación; además, con el distanciamiento temporal la comparación social con la fuente se vuelve menos saliente. Finalmente, la distinción entre influencia directa e indirecta subraya la relación entre la toma de posición expresada por los sujetos blanco y el carácter explícito de la alternativa minoritaria. En este sentido, la respuesta indirecta está referida a las posiciones no explícitas de la minoría, pero que deben inferirse de su discurso. Esta correspondencia implica que hay un mismo principio organizador, de orden más general, que estructura la respuesta directa e indirec- 
ta. Nos interesa formalizar esta relación de manera que nos permita establecer los límites en la definición de influencia indirecta.

En efecto, dado el conjunto de posiciones expresadas por la minoría (PM) y el conjunto de ítems por medio de los cuales se evalúa la influencia indirecta (IND), se define la relación indirecta de un ítem con las posiciones minoritarias como una función inyectiva del conjunto de ítems indirectos sobre el conjunto de posiciones explícitas de la minoría (ítems directos): IND $=\mathrm{f}(\mathrm{PM})$. Para comprender mejor esta relación definimos tres subconjuntos dentro del conjunto de ítems indirectos. $\mathrm{La}$ asignación de elementos al dominio de estos subconjuntos se hace de acuerdo con el criterio de idéntica estructura dentro de cada categoría temática de los ítems directos, pero no incluidos en el discurso minoritario, en el caso del subconjunto ítems Indirectos (I). Criterio de estructura complementaria y opuesta a los ítems directos, para el subconjunto de ítems Indirectos (II). $\mathrm{Y}$ en el subconjunto Indirectos (III) el criterio para su asignación es complementariedad y oposición a los ítems Indirectos (I), es decir, con una relación transitiva con las posiciones directamente expresadas por la minoría. Así pues, conjeturamos que un ítem indirecto está definido en base a una relación binaria no transitiva con el contenido del discurso minoritario.

De acuerdo con los postulados del modelo integrativo, una fuente minoritaria intragrupo plantea un conflicto menos intenso que una fuente categorizada como extragrupo. El sentimiento de identidad común permite una autoatribución de las características de la fuente que sobresalen en la situación preservando una identidad positiva en los sujetos. Sin embargo, la posición de los sujetos no es indiferente, los mismos juicios minoritarios pueden implicar una amenaza para la identidad de los sujetos ideológicamente próximos a la minoría (Mugny y Papastamou, 1982-83). Un conflicto de identificación es, pues, posible y puede ser contrario a la fuente de la misma categoría, o con una proximidad psicológica a los sujetos que generaría la necesidad de diferenciarse (Lemaine, 1975; 1984). Por otro lado, el inducir una percepción de la minoría como no representativa de su grupo lleva el conflicto a un polo interpersonal. El carácter individual de la fuente excluye la autoatribución de las características definidoras de una identidad social. Una minoría presentada como representativa, por el contrario, desliza el conflicto hacia el polo intergrupal, hacia una comparación social. En definitiva, una fuente representativa plantea un conflicto más fuerte que una fuente no representativa (Pérez y Mugny, 1985a).

En esta línea de análisis hipotetizamos, en términos de intensidad de conflicto, que la condición experimental en la que la minoría pertenece al intragrupo es representativa y además argumenta en contra de la identidad de los sujetos blanco, donde se da el máximo conflicto. De manera análoga, con una fuente de influencia categorizada como intragrupo y representativa, pero que argumenta a favor de los sujetos, el conflicto que crea es mínimo. Entre estas dos condiciones con una intensidad máxima y mínima se clasifican los restantes grupos experimentales de manera jerárquica. Una minoría extragrupo, que argumenta en contra de los sujetos a los que trata de influir, creará un conflicto de poca intensidad. Los sujetos no toman en consideración los juicios y opiniones de la minoría al establecerse un proceso de comparación social en el que resalta el carácter extragrupo de la fuente. Por el contrario, cuando una minoría extragrupo manifiesta una posiciones que son favorables a los sujetos, éstos pueden sentir amenazada su identidad ante una posible identificación espontánea con la minoría, el conflicto en este caso será más intenso. Finalmente, creemos que en las condi- 
ciones experimentales con una minoría sin categorizar y representativa, tanto si mantiene una posición favorable como contraria a la identidad de los sujetos blanco, el nivel de intensidad del conflicto es óptimo.

\section{METODO}

2.1. Sujetos. Un total de 214 alumnos de un Instituto de Enseñanza Media de Salamanca, cuyas edades oscilaban entre los 17 y 19 años, participaron en la experiencia. Una vez eliminados aquellos que no completaron alguna de las fases se retuvieron para el análisis 196 sujetos de ambos sexos, 87 varones y 109 mujeres, distribuidos aleatoriamente entre las distintas condiciones. El experimento se llevó a cabo en sus aulas habituales, durante las horas de clase y en ausencia de sus profesores. Todas las condiciones experimentales fueron presentadas en cada una de las clases.

2.2. Procedimiento. El experimento se desarrolló en dos fases consecutivas separadas por un intervalo de cuatro semanas. Durante la primera fase se indicaba a los sujetos que dentro del marco general del «Programa de Reforma de las Enseñanzas Medias» se estaban llevando a cabo un conjunto de estudios para conocer las opiniones de los alumnos. En concreto y en este momento, se les dijo: «nos interesa conocer vuestras opiniones respecto a la división de los estudios de bachillerato en ciencias y letras». Los sujetos expresaron sus opiniones sobre veinticuatro ítems de un cuestionario referidos a la problemática de los estudios de ciencias/letras (pretest). La segunda fase comenzaba con la lectura de un manifiesto atribuido a un grupo minoritario de estudiantes que mantenían una posición favorable a los estudios de letras. La introducción del texto minoritario se efectuó de formas diferentes; a una mitad de los sujetos se les presentaba el grupo minoritario como representativo de la opinión general de los alumnos; a la otra mitad se les presentó como no representativo de las opiniones del alumnado. Una vez terminada la lectura del manifiesto, texto inductor de influencia, los sujetos evaluaron la imagen de la fuente a través de un cuestionario formado por veinte escalas bipolares de adjetivos. El experimento finalizaba con la contestación al mismo cuestionario de opinión, sobre el tema de la división de los estudios del bachillerato, de la fase inicial (postest).

2.3. Diseño. La operacionalización de varios niveles de conflicto social se realizó por medio de la manipulación de dos variables independientes combinadas factorialmente en un diseño $3 \times 2$ (Categoría minoritaria $\times$ Representatividad de la fuente). El primer factor lo constituía la pertenencia categorial de la fuente minoritaria con tres niveles: Minoría de estudiantes de B.U.P. de Ciencias versus Minoría de estudiantes de B.U.P. de Letras versus Minoría de estudiantes de B.U.P. (sin identificar). El segundo factor estaba formado por la inducción de una percepción de la fuente de influencia en términos de representatividad con dos niveles: Minoría representativa versus Minoría no representativa. Para controlar la naturaleza de la relación intergrupal que se establece entre la fuente minoritaria y los sujetos blanco de influencia (intragrupo/extragrupo) se dividió la muestra de sujetos de acuerdo con el criterio identidad del alumno. Se establecen, de este modo, dos submuestras de sujetos: muestra de alumnos de letras $(\mathrm{N}=66)$ y muestra de alumnos de ciencias $(\mathrm{N}=130)$. 


\subsection{Material experimental}

2.4.1. Cuestionario de opinión. Este cuestionario está compuesto por 24 ítems en forma de proposiciones relativas a los estudios de ciencias o letras, que los sujetos debían valorar en una escala de intensidad Likert de 1 a 7 ( 1 = de acuerdo, 7 = en desacuerdo). Los ítems se organizan en torno a cuatro dimensiones definidas como Motivaciones, Inteligencia, Facilidad y Resultados. La primera dimensión agrupa ítems que hacen referencia a las razones de los alumnos para optar por los estudios de ciencias o de letras (ejemplo: El prestigio social que tienen las carreras de ciencias es lo que hace que sean más elegidas por los alumnos que las de letras). Los ítems de la dimensión Inteligencia aluden a las capacidades intelectuales de los alumnos que cursan uno u otro tipo de estudios (ejemplo: Normalmente se considera que los alumnos que cursan uno u otro tipo de estudios (ejemplo: Normalmente se considera que los alumnos que estudian ciencias tienen un C.I. elevado). Dentro de la dimensión Facilidad, los ítems argumentan sobre las ventajas de estudiar ciencias o de letras (ejemplo: Los estudios de ciencias poseen una complejidad mayor que los estudios de letras). Por último, los ítems de la dimensión Resultados son un conjunto de afirmaciones relativas a los rendimientos que obtienen los alumnos de ciencias y letras respectivamente (ejemplo: Los estudiantes de letras obtienen más becas y ayudas que los estudiantes de ciencias). Cada dimensión está integrada por 6 ítems, existiendo una simetría 3 a 3, correspondiendo las proposiciones en unos casos a ciencias y en otros a letras (ejemplo: El ítem «Los alumnos que más esfuerzo se exigen a sí mismos son aquellos que optan por estudios de ciencias.», encuentra su simétrico en el ítem «Los alumnos que eligen estudios de letras se exigen a sí mismos más esfuerzo que los que eligen ciencias.»). Los 24 ítems fueron distribuidos al azar dentro del cuestionario.

En las investigaciones sobre influencia minoritaria se distinguen normalmente dos índices de influencia. Un índice directo que se extrae a partir de los ítems del cuestionario de opinión empleados de manera coordinada como argumentos en el discurso de la minoría (ítems directos), y un índice indirecto calculado con los ítems del cuestionario de opinión que no aparecen en el texto minoritario inductor de influencia. En nuestro estudio manejamos dichos índices, pero el índice de influencia indirecta se descompone en tres subíndices:

a) Indice indirecto (I) estimado a través de los ítems del cuestionario de opinión que no se recogen en el panfleto minoritario. Pertenecen a cada una de las dimensiones temáticas y tiene una estructura idéntica a los ítems directos.

b) Indice indirecto (II). La estructura de los ítems en base a los cuales se calcula es complementaria y opuesta a los ítems directos en cada dimensión temática.

c) Por último, se computa un índice de influencia indirecta (III) con los ítems formulados de manera complementaria y opuesta a los ítems indirectos (I).

2.4.2. Texto minoritario. El intento de influencia se ejerció por medio de la lectura de un manifiesto atribuido a un grupo de estudiantes de B.U.P. El texto está organizado en tres cuerpos; en la parte superior y subrayado tipográficamente, se identifican a los autores como un grupo de estudiantes de B.U.P. 
de ciencias, letras o como un grupo de estudiantes de B.U.P. solamente, sin identificar otra pertenencia categorial. El segundo cuerpo del texto es una introducción encabezada por el título: «Comentarios ante los planes de estudios: Ciencias/Letras», donde se plantea el tema de los planes de estudio y las diferencias que origina cursar asignaturas de ciencias o de letras durante el bachillerato. La tercera parte del texto está formada por cuatro bloques, numerados correlativamente del I al IV, en los cuales el grupo minoritario aboga sistemáticamente en favor de los estudios de letras utilizando como argumentos dos ítems de cada una de las dimensiones del cuestionario de opinión - Motivaciones, Inteligencia, Facilidad y Resultados- reproducidos de manera literal.

2.4.3. Texto inductor de representatividad. Previamente a la lectura del manifiesto minoritario se administraba a los sujetos una consigna (subrayado y entre paréntesis lo que cambia según la condición experimental) en la cual se indicaba que el texto que a continuación iban a leer estaba redactado colectivamente (individualmente) por un grupo de alumnos de B.U.P. de un instituto similar al suyo y representa (no representa) bien la opinión general. Es decir, a la mitad de los sujetos se les inducía una percepción de los autores del texto como representativos de su categoría y en los sujetos restantes, por el contrario, se suscitaba una percepción de la minoría en términos de no representatividad de las opiniones de su categoría.

2.4.4. Cuestionario de representación social de la fuente. La representación social que los sujetos formaron de la fuente de influencia fue medida a través de veinte escalas bipolares de adjetivos. Los sujetos calificaron de 1 a 7 los adjetivos según creyeran que les correspondían a los autores del texto minoritario. Los términos incluidos en estas escalas proceden de las investigaciones previas sobre los procesos de influencia social (Papastamou, 1986; Pérez y Mugny, 1985b; Ricateau, 1970-71). Los ítems incluidos en el cuestionario se agrupan en varias dimensiones (el polo izquierdo entre paréntesis). Dimensión consistencia (Moscovici, 1976), formada por los ítems: coherentes (incoherentes), inseguros (seguros) y lógicos (ilógicos). Dimensión credibilidad (Hollander, 1960): incompetentes (competentes), creibles (increibles) y no dignos de fe (dignos de fe). Dimensión flexibilidad (Mugny, 1975): tolerantes (intolerantes), no dogmáticos (dogmáticos), flexibles (rígidos), abiertos (con prejuicios) y democráticos (autoritarios). Dimensión autonomía (Nemeth y Wachtler, 1974): dependientes (independientes), individualistas (colectivos) y representativos (no representativos). Dimensión moralidad: inmorales (morales) y generosos (egoístas). Las cuatro escalas bipolares restantes estaban formadas por los siguientes adjetivos referidos a una consistencia y credibilidad posicional (Mugny y Pérez, 1985): irrealistas (realistas); sin objetividad (objetivos); críticos (acríticos) y desinteresados (guiados por un interés). Tanto los ítems dentro del cuestionario, como los adjetivos en los polos de cada escala fueron distribuidos aleatoriamente.

\section{RESULTADOS}

3.1. Influencia Directa. Examinemos, en primer lugar, los resultados obtenidos en relación con el índice de influencia directa. Este índice se estimó calculando la media de las diferencias en las puntuaciones entre el pretest y el 
TABLA I

Medias de cambio de opinión en los ítems directos del cuestionario de opinión (desviación típica entre paréntesis) y análisis de varianza de la muestra de sujetos de ciencias.

\begin{tabular}{|c|c|c|c|c|c|}
\hline & \multicolumn{2}{|c|}{ Representativa } & \multicolumn{3}{|c|}{ No representativa } \\
\hline Minoría letras & \multicolumn{2}{|c|}{$\begin{array}{c}+0.069(0.61) \\
n=23\end{array}$} & \multicolumn{3}{|c|}{$\begin{array}{c}-0.125(0.80) \\
\mathrm{n}=22\end{array}$} \\
\hline Minoría ciencias & \multicolumn{2}{|c|}{$\begin{array}{c}-0.603(0.90) \\
n=23\end{array}$} & \multicolumn{3}{|c|}{$\begin{array}{c}-0.167(0.99) \\
\mathrm{n}=21\end{array}$} \\
\hline Minoría sin identificar & \multicolumn{2}{|c|}{$\begin{array}{c}+0.188(0.91) \\
n=18\end{array}$} & \multicolumn{3}{|c|}{$\begin{array}{c}-0.533(0.62) \\
n=23\end{array}$} \\
\hline \multicolumn{6}{|c|}{ Análisis de varianza } \\
\hline Fuente & S.C. & $\mathrm{gl}$ & M.C. & $\mathrm{F}$ & $\mathrm{p}$ \\
\hline Minoría (A) & 2.862 & 2 & 1.431 & 2.20 & ns \\
\hline Representatividad (B) & 0.818 & 1 & 0.818 & 1.26 & ns \\
\hline$A \times B$ & 7.080 & 2 & 3.540 & 5.46 & 0.005 \\
\hline Error & 80.469 & 124 & 0.649 & & \\
\hline
\end{tabular}

postest, de los 8 ítems directos del cuestionario de opinión, para cada sujeto. En los ANOVAS construidos (Categoría minoritaria x Representatividad de la fuente) solamente aparece un efecto de interacción significativo en la muestra de sujetos de ciencias $\left[\mathrm{F}_{2,124}=5.46, \mathrm{p}<0.005\right]$. La tabla 1 a $^{\mathrm{a}}$ recoge el cuadro de medias y análisis de varianza. La descomposición de esta interacción refleja que una minoría de letras representativa se diferencia significativamente de una minoría sin identificar no representativa $(t=3,31, \mathrm{p}<0.001)$. Así mismo, hay diferencias significativas entre la influencia obtenida por una minoría sin identificar presentada como representativa y no representativa $(t=2.88, p<0.007)$. De acuerdo con estos resultados puede afirmarse que precisar la pertenencia categorial de una fuente minoritaria, intragrupo o extragrupo, induce un conflicto de naturaleza intergrupal que determina el nivel de la influencia obtenida. Para una minoría intragrupo mantener una posición contraria a su grupo supone un aumento del coste social, que impide toda identificación con sus posiciones, máxime si es representativa. En este sentido, hay diferencias significativas entre una minoría de ciencias con una minoría de letras $(t=2.96, p<0.005)$ y con una minoría sin identificar, siendo ambas representativas $(t=-2.78$, $\mathrm{p}<0.008$ ). Pero si la pertenencia categorial no se explicita, la variable responsable de los cambios obtenidos es la representatividad de la fuente.

3.2. Influencia Indirecta. Recordamos que hemos calculado cuatro índices de influencia indirecta. Un índice de influencia indirecta (I) que resulta de la media de las diferencias, para cada sujeto, en las puntuaciones dadas en el pretest y el postest a los 4 ítems indirectos (I) del cuestionario de opinión. Los índices de influencia (II) y (III) se computan de manera análoga, pero para mantener un sentido unívoco en la interpretación del signo se extraen las medias de las diferencias de las puntuaciones entre el postest y el pretest de los 8 y 4 ítems indirectos (II) y (III) del cuestionario de opinión respectivamente. Además, se calculó un índice de influencia indirecta global con los 16 ítems indirectos del cuestionario. 
El estudio de la influencia indirecta global no refleja ningún efecto significativo en la muestra de sujetos de ciencias. Sí aparece, por el contrario, un efecto de la variable categoría de la fuente minoritaria en el Anova de la muestra de sujetos de letras $\left[\mathrm{F}_{2,60}=2.94, \mathrm{p}<0.006\right]$. En la tabla $2 .^{\mathrm{a}}$ se exponen los resultados de esta muestra. El peso más importante en esta significación procede de la diferencia entre la influencia negativa que obtienen las fuentes extragrupo, minorías de ciencias $(m=-0.093)$, con las minorías sin identificar $(m=+0.291$; $t=-2.57, p<0.01)$. Esta diferencia se mantiene solamente en el nivel representativo $(t=-2.75, p<0.01)$. No son significativas, sin embargo, las diferencias existentes entre una fuente intragrupo y una fuente de influencia extragrupo en ninguna de las condiciones de representatividad operacionalizadas.

\section{TABLA II}

Medias de cambio de opinión en los items indirectos del cuestionario de opinión y análisis de varianza de la muestra de sujetos de letras.

\begin{tabular}{|c|c|c|c|c|c|}
\hline & \multicolumn{2}{|c|}{ Representativa } & \multicolumn{3}{|c|}{ No representativa } \\
\hline Minoría letras & \multicolumn{2}{|c|}{$\begin{array}{c}-0.050(0.49) \\
n=12\end{array}$} & \multicolumn{3}{|c|}{$\begin{array}{c}+0.253(0.56) \\
n=12\end{array}$} \\
\hline Minoría ciencias & \multicolumn{2}{|c|}{$\begin{array}{c}-0.234(0.30) \\
\mathrm{n}=11\end{array}$} & \multicolumn{3}{|c|}{$\begin{array}{c}+0.047(0.54) \\
n=10\end{array}$} \\
\hline Minoría sin identificar & \multicolumn{2}{|c|}{$\begin{array}{c}+0.323(0.62) \\
n=12\end{array}$} & \multicolumn{3}{|c|}{$\begin{array}{c}+0.259(0.45) \\
n=9\end{array}$} \\
\hline \multicolumn{6}{|c|}{ Análisis de varianza } \\
\hline Fuente & S.C. & gl & M.C. & $\mathrm{F}$ & $\mathrm{p}$ \\
\hline Minoría (A) & 1.535 & 2 & 0.767 & 2.94 & 0.06 \\
\hline Representatividad (B) & 0.490 & 1 & 0.490 & 1.88 & ns \\
\hline$A \times B$ & 0.451 & 2 & 0.225 & 0.86 & ns \\
\hline Error & 15.681 & 60 & 0.261 & & \\
\hline
\end{tabular}

$\mathrm{El}$ análisis de los datos referidos al indice de influencia indirecta (I) revela efectos significativos en ambas muestras de sujetos; la tabla $3 .^{a}$ presenta los resultados obtenidos con este índice de influencia. En relación con la muestra de sujetos de letras aparece como significativa la variable representatividad de la fuente $\left[F_{1,60}=6.22, p<0.01\right]$. En general, cuando la posición de la fuente minoritaria corresponde con una percepción individual, no representativa, es menos aceptada $(\mathrm{m}=-0.404)$ que cuando es percibida como representativa de las opiniones del grupo $(\mathrm{m}=+0.417 ; \mathrm{t}=2.61, \mathrm{p}<0.01)$. La interpretación de los resultados obtenidos en el análisis de varianza de la muestra de sujetos de ciencias es más compleja al aparecer, simultáneamente, una interacción entre las variables categoría y representatividad de la fuente de influencia $\left[F_{2}\right.$, $124=3.34, \mathrm{p}<0.03]$ y una tendencia referida al efecto de la variable categoría de la fuente $\left[\mathrm{F}_{2,124}=2.37, \mathrm{p}<0.09\right]$; el nivel de significación alcanzado es menor y sólo permite considerarlo como una tendencia en los datos. Puede afirmarse, no obstante, que la representatividad está relacionada con la influencia indirecta (I) obtenida; en otros términos, una fuente que no destaca su pertenencia categorial, minoría sin identificar, tiene un impacto mayor si es presentada como representativa de las opiniones de su grupo que si es percibida como 


\section{TABLA III}

Medias de cambio de opinión en los ítems indirectos (I) del cuestionario de opinión y análisis de varianza con la muestra de sujetos de ciencias y letras.

\begin{tabular}{|c|c|c|c|c|c|c|c|c|c|c|}
\hline \multirow{2}{*}{\multicolumn{3}{|c|}{$\begin{array}{l}\text { Minoría / Representatividad } \\
\text { Sujetos ciencias }\end{array}$}} & \multicolumn{8}{|c|}{ Sujetos letras } \\
\hline & & & $N$ & & & & $\mathrm{~N}$ & & & \\
\hline \multirow{6}{*}{\multicolumn{3}{|c|}{$\begin{array}{l}\text { Minoría letras / Representativa } \\
\text { Minoría letras / No representativa } \\
\text { Minoría ciencias / Representativa } \\
\text { Minoría ciencias / No representativa } \\
\text { Minoría sin ident. / Representativa } \\
\text { Minoría sin ident. / No representativa } \\
\end{array}$}} & 12 & \multirow{6}{*}{\multicolumn{3}{|c|}{$\begin{array}{l}+0.271(0.91) \\
-0.496(1.64) \\
+0.205(1.78) \\
-0.550(1.38) \\
+0.777(1.14) \\
-0.167(0.68)\end{array}$}} & 23 & \multirow{6}{*}{\multicolumn{3}{|c|}{$\begin{array}{l}+0.109(0.81) \\
+0.057(0.97) \\
-0.478(1.15) \\
-0.274(1.03) \\
+0.319(0.73) \\
-0.554(1.14)\end{array}$}} \\
\hline & & & 12 & & & & 22 & & & \\
\hline & & & 11 & & & & 23 & & & \\
\hline & & & 10 & & & & 21 & & & \\
\hline & & & 12 & & & & 18 & & & \\
\hline & & & 9 & & & & 23 & & & \\
\hline \multicolumn{2}{|l|}{$\begin{array}{l}\text { Análisis } \\
\text { de Varianza }\end{array}$} & \multicolumn{4}{|c|}{ Sujetos letras } & \multicolumn{5}{|c|}{ Sujetos ciencias } \\
\hline Fuente & S.C. & $\mathrm{gl}$ & M.C. & $\mathrm{F}$ & $\mathrm{p}$ & S.C. & $\mathrm{gl}$ & M.C. & $\mathrm{F}$ & \\
\hline Minoría (A) & 2.806 & 2 & 1.403 & 0.80 & ns & 4.646 & 62 & 2.348 & 2.37 & 0.09 \\
\hline Representatividad (B) & 10.951 & 11 & 10.951 & 6.22 & 0.01 & 1.864 & 41 & 1.864 & 1.26 & $\mathrm{~ns}$ \\
\hline$A \times B$ & 0.110 & 2 & 0.055 & 0.03 & ns & 6.611 & 12 & 3.306 & 5.46 & 0.03 \\
\hline Error & 105.719 & 60 & 1.762 & & & 122.742 & 2124 & 0.990 & & \\
\hline
\end{tabular}

no representativa. En estas dos condiciones encontramos la influencia máxima y mínima con una diferencia claramente significativa $(t=2.97, p<0.005)$. La minoría categorizada como intragrupo logra una influencia menor que la minoría extragrupo, sobre todo si representan bien la opinión general del grupo $(t=2.00, p<0.05)$. Este patrón se mantiene, con un nivel de significación menor, cuando se induce una percepción de la fuente como no representativa $(t=1.69, p<0.09)$. Por último, cuando la fuente está categorizada como extragrupo la variable representatividad no entra en funcionamiento.

\section{TABLA IV}

Medias de cambio de opinión en los ítems indirectos (II) del cuestionario de opinión y análisis de varianza con la muestra de sujetos de ciencias.

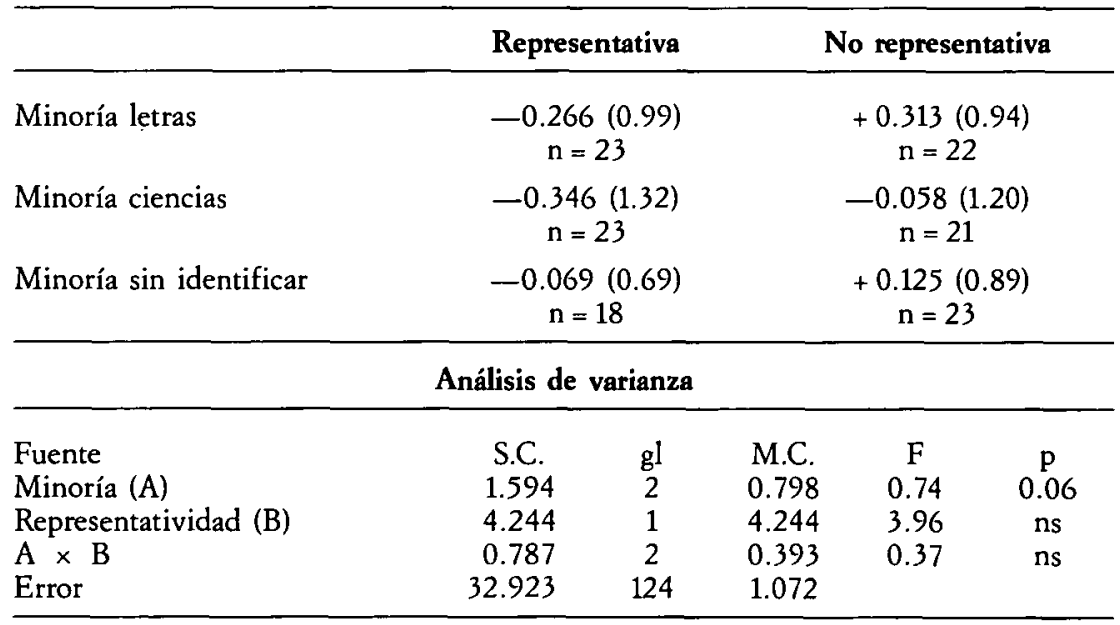


En cuanto a los resultados del indice de influencia indirecta (II) solamente encontramos un efecto significativo en la muestra de sujetos de ciencias debido a la variable representatividad de la fuente minoritaria $\left[\mathrm{F}_{1,124}=3.96, \mathrm{p}<0.04\right]$. La tabla $4{ }^{\text {a }}$ muestra los resultados obtenidos, medias y análisis de varianza, en esta muestra. Los grupos con el nivel de no representatividad alcanzan un mayor impacto que los grupos con el nivel de representatividad; sin embargo, sólo hay diferencias significativas entre las minorías de letras, extragrupo $(t=-2.01$, $\mathrm{p}<0.05$ ); no constatándose diferencias entre las minorías de ciencias, intragrupo $(t=-0.86$, ns.) o entre las minorías sin identificar $(t=-0.90$, ns. $)$.

Finalmente, los resultados obtenidos con el indice de influencia indirecto (III) no denotan ningún efecto significativo, tanto en la muestra de sujetos de letras como en la de sujetos de ciencias.

3.3. Representación social de la fuente minoritaria. Un análisis factorial de componentes principales con rotación varimax, aplicado a las respuestas del cuestionario de representación de la fuente de ambas muestras de sujetos, procesadas conjuntamente, pone en evidencia cinco factores que explican el 61.3 por 100 de la varianza total.

El primer factor es el más importante con un porcentaje de varianza explicada del 34 por 100 . Es un factor en el que saturan trece ítems del cuestionario de imagen de la fuente, referidos a la dimensión credibilidad: creibles $(+75)$,

\section{TABLA V}

Coeficientes factoriales del análisis de componentes principales del cuestionario de imagen de la fuente después de baber realizado una rotación ortogonal varimax $(I=196, J=20)$. Los coeficientes ban sido redondeados y multiplicados por 100, sustituyendo las cargas menores de 25 por ceros.

\begin{tabular}{lrrrrrrr}
\hline & \multicolumn{7}{c}{ FACTORES } \\
\cline { 2 - 8 } Items & c. & \multicolumn{1}{c}{ I } & II & III & IV & V & media \\
\hline Lógicos & 63 & +68 & +35 & 00 & 00 & 00 & 3.76 \\
Generosos & 54 & +60 & +38 & 00 & 00 & 00 & 4.67 \\
Incompetentes & 73 & -72 & 00 & 00 & +38 & 00 & 4.29 \\
No dogmáticos & 60 & 00 & 00 & -27 & +72 & 00 & 3.60 \\
Representativos & 63 & 00 & 00 & +75 & 00 & 00 & 4.28 \\
Creíbles & 71 & +75 & +35 & 00 & 00 & 00 & 3.79 \\
Dependientes & 56 & 00 & 00 & 00 & 00 & +70 & 4.43 \\
Irrealistas & 61 & -56 & -50 & 00 & 00 & 00 & 4.91 \\
Tolerantes & 57 & +48 & +31 & +34 & +35 & 00 & 4.31 \\
Flexibles & 52 & +27 & +39 & +39 & +36 & 00 & 4.75 \\
Democráticos & 60 & +73 & 00 & 00 & 00 & 00 & 4.29 \\
Sin objetividad & 52 & 00 & -55 & -34 & 00 & 00 & 3.64 \\
No dignos de fe & 59 & -47 & 00 & -51 & 00 & 00 & 3.97 \\
Individualistas & 51 & 00 & 00 & -67 & 00 & 00 & 3.31 \\
Críticos & 67 & 00 & 00 & 00 & 00 & -75 & 2.88 \\
Inseguros & 74 & -37 & -37 & 00 & +58 & +34 & 4.71 \\
Inmorales & 62 & 00 & -68 & 00 & 00 & 00 & 4.29 \\
Coherentes & 63 & +31 & +72 & 00 & 00 & 00 & 4.15 \\
Abiertos & 66 & +35 & +62 & 00 & +28 & 00 & 4.27 \\
Desinteresados & 67 & +50 & -34 & 00 & +49 & 00 & 5.19 \\
\hline \% Varianza total & & & & & & & \\
explicada & 61.3 & 33.97 & 10.48 & 6.16 & 5.60 & 5.09 & \\
\hline
\end{tabular}


incompetentes (-71) y no dignos de fe (-47); a la dimensión consistencia: lógicos $(+68)$, inseguros $(-37)$ y coherentes $(+31)$; a la dimensión flexibilidad: democráticos $(+73)$, tolerante $(+48)$, abierto $(+35)$ y flexibles $(+27)$, y a la dimensión moralidad: generosos $(+60)$. Encontramos, así mismo, dos ítems referidos a la credibilidad posicional: irrealistas $(-56)$ y desinteresados $(+50)$. En principio cabe pensar que este factor es de naturaleza evaluativa general, ya que incluye ítems de casi todas las dimensiones. Sin embargo, es preciso fijar con más nitidez los componentes que definen este factor para conocer su verdadera naturaleza.

En este sentido hay que hacer algunas precisiones: En primer lugar, considerando los ítems con peso superior a \pm 25 , encontramos ítems con cargas factoriales en tres e incluso cuatro ejes - desinteresados, abiertos, no dignos de $\mathrm{fe}$, inseguros, no dogmáticos, tolerantes y flexibles-. Entendemos que en el momento de interpretar un factor es preciso considerar estos ítems como menos específicos valorándolos sólo en el factor en el que presentan el peso máximo. En segundo lugar, hay que hacer mención especial de los ítems - flexibles y tolerantes-, ambos de la dimensión flexibilidad, que tienen pesos factoriales cercanos a +35 en los factores I, II, III y IV. Si tenemos en cuenta lo anteriormente expresado, el primer factor, aun siendo de naturaleza evaluativa, se centraría en la valoración de la credibilidad de la fuente, evaluación que no es posible sin estimar la consistencia, del estilo de negociación de la moralidad.

$\mathrm{Al}$ igual que el primer factor de naturaleza evaluativa general, el segundo factor forma un constructo alrededor de la percepción evaluativa de la consistencia, que los sujetos valoran en su relación con el estilo de negociación y la moralidad. Explica el 10.48 por 100 de la varianza total y lo integran fundamentalmente los ítems: coherentes $(+72)$, inseguros $(-37)$ y lógicos $(+35)$ de la dimensión consistencia; abiertos $(+62)$, tolerantes $(+31)$ y flexibles $(+39)$ de la dimensión flexibilidad; inmorales $(-68)$ y generosos $(+38)$ de la dimensión moralidad; creibles $(+35)$ de la dimensión credibilidad y desinteresados $(-34)$, sin objetividad $(-55)$ e irrealistas $(-50)$ relacionados con una consistencia y credibilidad posicional.

En el eje que forma el tercer factor saturan altamente dos ítems de la dimensión autonomía: representativos $(+75)$ e individualistas $(-67)$. Dado que los ítems de esta dimensión no saturan en ningún otro factor y que los ítems restantes, flexibles $(+39)$, tolerantes $(+34)$, no dogmáticos $(-27)$, no dignos de fe $(-51)$ y sin objetividad (-34), están afectados por las precisiones anteriormente expuestas, tienen pesos superiores a \pm 25 en tres factores simultáneamente, podemos afirmar que este factor está referido a la autonomía minoritaria. (Mugny y Pérez, 1985; Papastamou, 1986).

Por medio del cuarto factor se describe el estilo de negociación, flexible/rígido, puesto en juego por la minoría. El núcleo de este factor, que explica el 5.60 por 100 de la varianza total, está formado por ítems de la dimensión flexibilidad: no dogmáticos $(+72)$, flexibles $(+36)$, tolerantes $(+35)$ y abiertos $(+28)$. No obstante, el estilo de negociación adoptado por la minoría afecta a la consistencia y credibilidad, por lo que en este factor aparecen ítems de las dos dimensiones y, también, ítems relacionados con una consistencia y credibilidad posicional: inseguros $(+58)$, incompetentes $(+38)$ y desinteresados $(+49)$.

El quinto y último factor está formado por tres ítems y explica el 5.09 por 100 de la varianza total. La fuente minoritaria es descrita como crítica $(-75)$, dependiente $(+70)$ e insegura $(+47)$. 
Veamos ahora en qué medida la representación social que los sujetos blanco de influencia elaboran de la fuente minoritaria permite comprender las diferencias en el impacto del mensaje sobre la estructura de las opiniones. Señalemos que el análisis de varianza (Identidad de sujeto $\times$ Categoría de la fuente $\times$ Representatividad) de las puntuaciones factoriales, para los cinco factores obtenidos con el cuestionario de representación social de la fuente pone de manifiesto diferencias significativas entre condiciones experimentales en los factores I, II y IV, como muestra la tabla 6. .

De manera general, hay diferencias significativas entre la imagen de la fuente de influencia que tienen los sujetos según su identidad. Los sujetos de la muestra de ciencias describen a la fuente minoritaria como más creible, competente, lógica, democrática y desinteresada [Factor $\mathrm{I}: \mathrm{F}_{1,184}=66.868, \mathrm{p}<0.001$ ], que

\section{TABLA VI}

Puntuaciones factoriales medias para los Factores I, II y IV del cuestionario de representación social de la fuente minoritaria y resultados del análisis de varianza (ANOVA $2 \times 2 \times 3$ ).

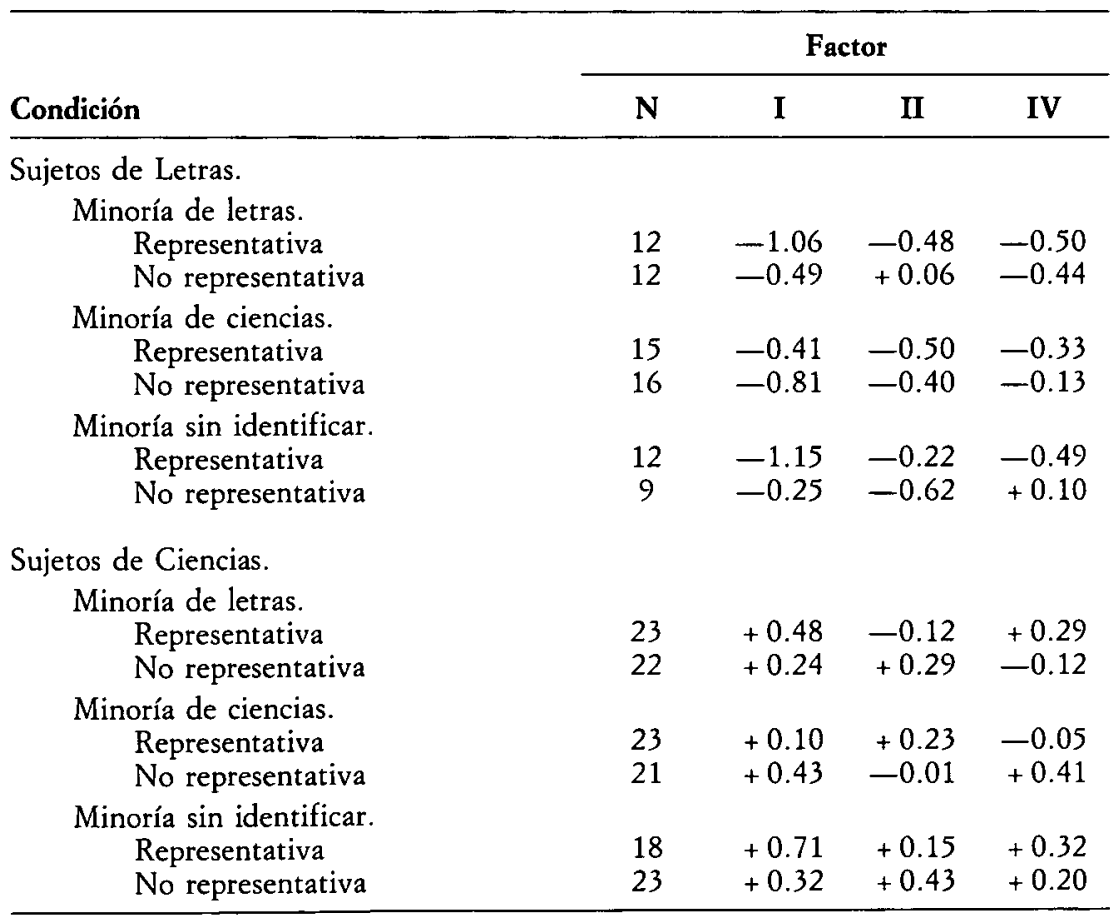

Análisis de varianza

Fuente

Identidad de los sujetos (A)

Categoría minoritaria (B)

Representatividad (C)

$A \times B$

$\mathrm{A} \times \mathrm{C}$

$\mathrm{B} \times \mathrm{C}$

$\mathrm{A} \times \mathrm{B} \times \mathrm{C}$

Error

$\begin{array}{cccccc}\mathrm{F} & \mathrm{p} & \mathrm{F} & \mathrm{p} & \mathrm{F} & \mathrm{p} \\ 66.868 & 0.001 & 11.603 & 0.001 & 11.098 & 0.001 \\ 0.192 & \mathrm{~ns} & 0.306 & \mathrm{~ns} & 0.646 & \mathrm{~ns} \\ 0.248 & \mathrm{~ns} & 1.037 & \mathrm{~ns} & 0.234 & \mathrm{~ns} \\ 1.669 & \mathrm{~ns} & 0.739 & \mathrm{~ns} & 0.221 & \mathrm{~ns} \\ 3.740 & 0.05 & 0.055 & \mathrm{~ns} & 1.184 & \mathrm{~ns} \\ 0.090 & \mathrm{~ns} & 1.631 & \mathrm{~ns} & 1.840 & \mathrm{~ns} \\ 4.936 & 0.008 & 1.023 & \mathrm{~ns} & 0.850 & \mathrm{~ns} \\ & & & & & \end{array}$


los sujetos de la muestra de letras. Así mismo, hay diferencias significativas en el Factor II $\left(F_{1,184}=11.603, p<0.001\right)$, los sujetos de ciencias perciben a la minoría como más consistente, coherente, abierta y generosa que los sujetos de letras. Por último, en el Factor IV las diferencias tienen el mismo sentido $\left[\mathrm{F}_{1}\right.$, $184=11.098, \mathrm{p}<0.001]$. La fuente minoritaria de influencia es, para los sujetos de ciencias, menos dogmática y más flexible que para los sujetos de la muestra de letras.

En relación con el Factor I, referido a una evaluación general estructurada alrededor de la credibilidad, aparece una interacción de primer orden $\left[\mathrm{F}_{1}\right.$ $184=3.740, \mathrm{p}<0.05$ ] entre la identidad de los sujetos y la representatividad y una interacción de segundo orden entre la identidad de los sujetos, la categorización y la representatividad de la fuente $\left[\mathrm{F}_{2,184}=4.936, \mathrm{p}<0.008\right]$. La elaboración de la imagen de la minoría parece responder a un patrón común pero con diferente sentido de acuerdo con la identidad de los sujetos blanco. La inducción de una percepción de la minoría como no representativa lleva a los sujetos de la muestra de ciencias a elaborar una imagen de la fuente caracterizada por la atribución de mayor credibilidad, siempre y cuando la minoría no esté categorizada como extragrupo. Los sujetos de la muestra de letras se comportan de manera análoga, pero en sentido contrario; la representatividad mejora la evaluación de la imagen de la fuente minoritaria, menos para una fuente extragrupo.

\section{DISCUSION}

Los procesos de influencia minoritaria, en cuanto regulados por mecanismos psicosociales, no son unidimensionales, monolíticos o estáticos. Dada esta complejidad, el presente trabajo cumple su objetivo como una primera aproximación experimental al estudio de las relaciones entre la intensidad del conflicto social y la influencia obtenida por una minoría. No obstante, los resultados permiten adelantar algunas conclusiones.

En primer lugar, la jerarquía de grupos experimentales que formulamos ha de ser interpretada en base a los resultados obtenidos. En efecto, la curva resultante de la hipótesis de la función cuadrática entre intensidad del conflicto e impacto social que plantean Mugny y Pérez $(1985 ; 1986 ; 1987)$ es esencialmente heurística; situando el problema en determinar la significación que caracteriza los diferentes puntos de inflexión. En este sentido, el ajuste de nuestros resultados a la hipotética curva es aceptable como muestran las Figuras $1 .^{\mathrm{a}} \mathrm{y}$ $2 .^{a}$ en las que se representa gráficamente las curvas de influencia en función de la intensidad del conflicto.

De manera general los resultados apoyan la hipótesis según la cual un conflicto máximo y un conflicto mínimo disminuyen drásticamente la influencia de las minorías. La interpretación de esta jerarquía debe hacerse diferenciando las respuestas de los sujetos según la muestra a la que pertenecen. Así, en relación con la muestra de sujetos de ciencias una minoría intragrupo y representativa que argumenta en contra de la identidad de los sujetos crea un conflicto de una intensidad máxima que anula todo su potencial de influencia. No podemos afirmar que las respuestas expresadas, tanto a nivel directo como a nivel indirecto, ante una minoría con la misma pertenencia categorial que los sujetos blanco sean tributarias de un favoritismo intragrupo como se afirma desde un 


\section{FIGURA I}

Representación gráfica de la influencia minoritaria en función de la intensidad del conflicto en la muestra de sujetos de ciencias.

$\checkmark$ Influencia directa.

- influencia indirecta (I).

$\Delta$ Influencia indirecta (II).

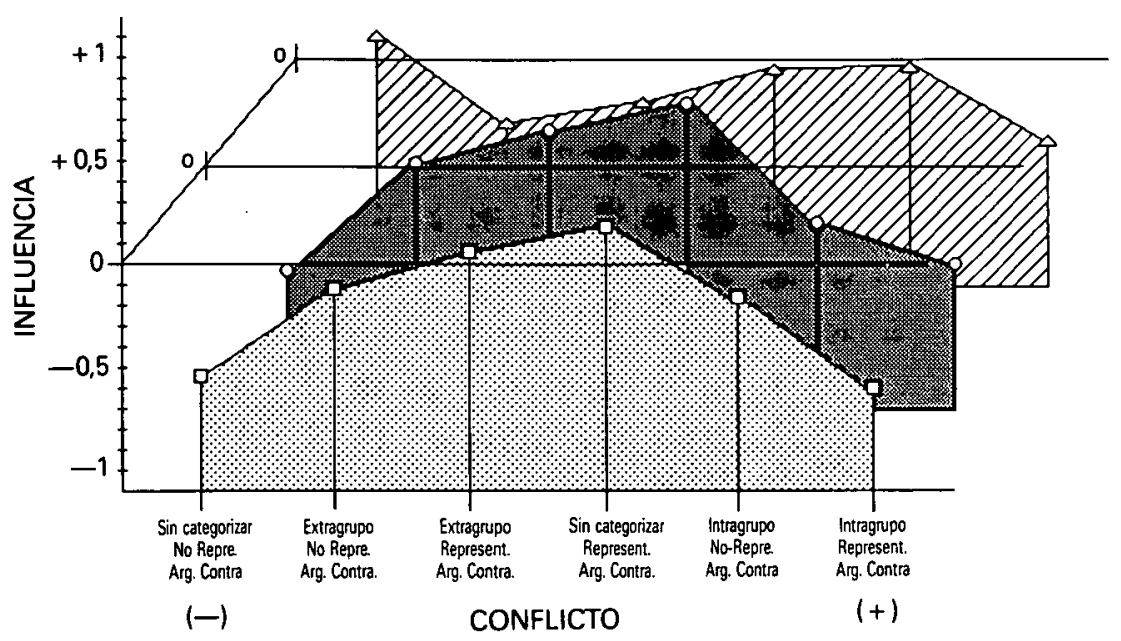

modelo intergrupo de la influencia minoritaria (Tajfel, 1978; Tajfel y Turner, 1979; Turner, 1984). Cabe pensar que la pertenencia a una categoría social común entre la minoría y los sujetos blanco conlleva un fuerte conflicto de identificación. En este sentido, el contenido mismo de los juicios y opiniones de la minoría supone una amenaza para la identidad de los sujetos. No se excluiría que la fuente intragrupo sea sometida a una discriminación. Los sujetos efectuarían una división categorial nueva, dentro de la propia categoría entre «nosotros» y «ellos», que tendría la función de proteger la identidad social. La minoría intragrupo es vista como «otros», una suerte de extragrupo (Zavalloni, 1971; Zavalloni y Louis-Guerin, 1984). En otros términos, una fuente categorizada como intragrupo puede inducir un conflicto de identificación y ser rechazada (Mugny, Kaiser y Papastamou, 1983; Pérez y Mugny, 1985b). La minoría extragrupo obtiene, sin embargo, influencia a nivel directo e indirecto a pesar de estar sometida a una discriminación, apareciendo un efecto paradójico (Aebischer, Hewtone y Henderson, 1984; Pérez y Mugny, 1986, 1987). Por otro lado, el hecho de que la significación estadística en las respuestas directas aparezca sólo en la muestra de ciencias nos lleva a pensar que para entender la influencia a nivel directo es fundamental considerar las tendencias en la población «zeitgeist» (Maass, Clark y Haberkom, 1982). Por último, la interacción revela que la influencia obtenida guarda relación directa con la representatividad de la fuente cuando no se explicita la pertenencia categorial, en suma, cuando el conflicto no es de naturaleza intergrupal.

El ajuste de la tasa de influencia obtenida a la curva hipotética es más precario en la muestra de sujetos de letras, sobre todo con el índice de influencia indirecta (I). En este caso la variable explicativa de los resultados es la represen- 


\section{1}

tatividad. Una minoría intragrupo que argumenta en favor de la propia identidad de los sujetos blanco de influencia y representativa crea un conflicto mínimo, los sujetos pueden pensar que la minoría mantiene esa posición guiada por su propio interés disminuyendo su impacto. De la misma manera, ante una minoría extragrupo representativa, que expone un punto de vista favorable a los sujetos blanco, el conflicto generado es más intenso al aumentar la relevancia de los costes sociales que implica una posible identificación (Mugny, Ibáñez y col., 1986). Tal como predecimos es la minoría sin categorizar y representativa la que obtiene mayor influencia. La percepción de la fuente en términos de no representatividad impide la influencia tanto para una minoría intragrupo como para una minoría extragrupo o sin categorizar. Sin duda este hecho no es ajeno a la imagen que los sujetos elaboran en esta muestra, como veremos más adelante.

\section{FIGURA II}

Representación gráfica de la influencia minoritaria en función de la intensidad del conflicto en la muestra de sujetos de letras.

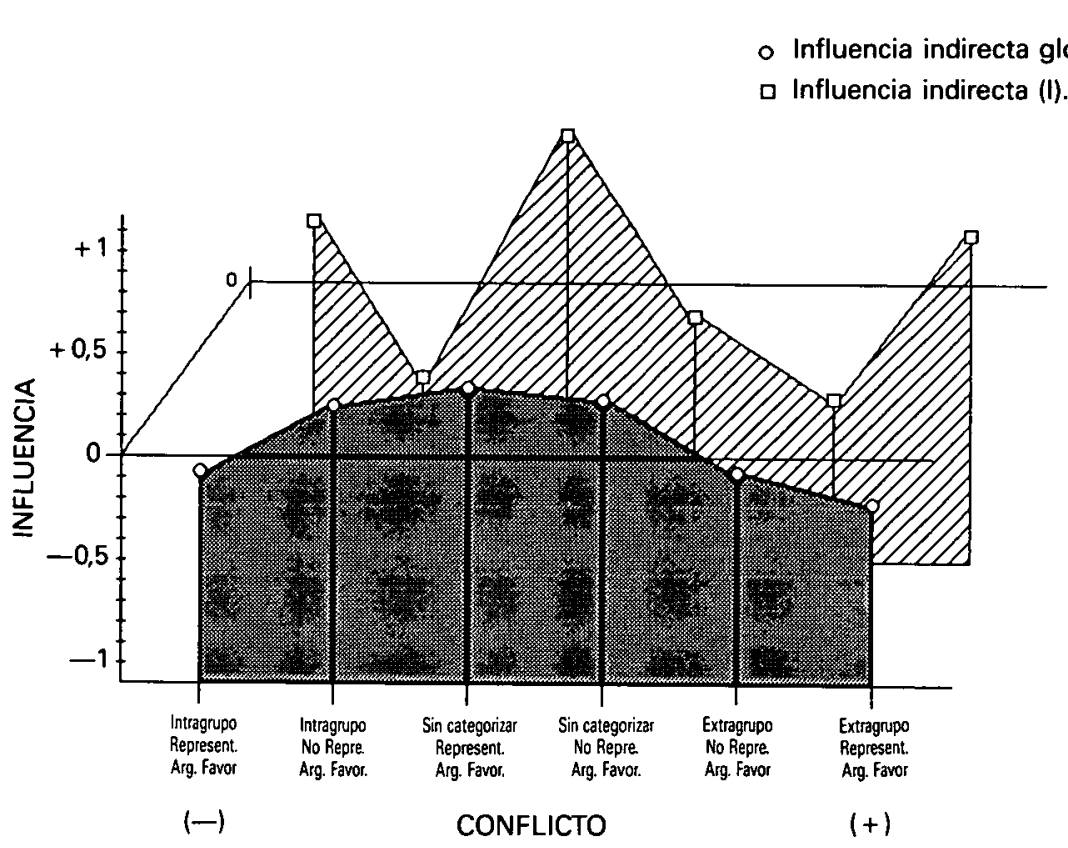

En segundo lugar, los resultados de los índices de influencia indirecta ponen de relieve la necesidad de fijar los límites de la definición de influencia indirecta. En el modelo integrativo Mugny y Pérez (1986) agrupan bajo el denominador común de influencia indirecta índices de influencia tales como influencia subyacente o influencia privada, pues responden al mismo mecanismo. Nuestro trabajo demuestra que, si bien todos los índices de influencia indirecta considerados obedecen a un proceso común, existen diferencias que señalan los límites 
de la influencia indirecta. En este sentido, se obtienen efectos significativos al considerar el índice (II), de naturaleza similar a los utilizados en las investigaciones de la Escuela de Ginebra, y en el índice (I), cuyos ítems guardan una relación binaria con los defendidos en el texto por la minoría, pero no con el índice de influencia (III), cuyos ítems están formulados en base a una relación transitiva con las posiciones defendidas por la minoría. En síntesis, un ítem indirecto estaría definido por la relación binaria no transitiva con el contenido del texto inductor de influencia.

En cuanto a la influencia indirecta los resultados confirman, de nuevo, la hipótesis del conflicto óptimo que, como consecuencia de la aparición de una alternativa consistente y representativa, obtiene el impacto mayor. Son los grupos con una minoría sin categorizar los que presentan los índices de influencia a nivel indirecto más elevados. Es necesario precisar, no obstante, que en el contexto social tal como se lo representa el sujeto no hay una división binaria -intragrupo/extragrupo- sino multidimensional, con categorizaciones cruzadas (Deschamps, 1977). Los grupos experimentales en los que la minoría no era identificada como de ciencias o letras pueden ser categorizados por los sujetos blanco como estudiantes, formando de este modo un intragrupo. Así mismo, la variable representatividad de la fuente actúa de acuerdo con las hipótesis formuladas, especialmente cuando no opera un conflicto de identificación categorial. Esta influencia indirecta puede explicarse desde los postulados del modelo integrativo propuesto por la Escuela de Ginebra (Mugny y Pérez, 1986), cuyo origen es un principio de validación que procede de la retranscripción psicológica del conflicto social al nivel de las representaciones de la totalidad del campo de innovación. Una actividad cognitiva que implica la recategorización de las entidades sociales implicadas, la redefinición de los atributos pertenecientes a esas categorías y la reelaboración de los contenidos del mensaje.

Finalmente, puede afirmarse que la imagen social de la fuente constituye, sin duda, un mediador de la influencia a través de las notables modificaciones que la representación social de la minoría sufre en la relación del sujeto con la fuente. Los sujetos blanco de influencia llevan a cabo una reestructuración de la representación social de la minoría en torno a la dimensión credibilidad, consistencia y estilo de negociación, que dan cuenta, en parte, de la acción de las variables independientes observadas a nivel de la influencia ejercida por una minoría. El primer eje, de carácter básicamente evaluativo, incluye la dimensión credibilidad, consistencia y estilo de negociación de la fuente. Además de este primer eje general, la representación de una fuente de influencia minoritaria está integrada por dos ejes autónomos referidos a la consistencia y al estilo de negociación.

Los datos muestran una imagen de la minoría marcadamente negativa, independientemente de la condición experimental, en la muestra de sujetos de letras y positiva en la muestra de sujetos de ciencias. Dos aspectos hay que destacar en estos resultados; por un lado, la minoría intragrupo no obtiene influencia a pesar de tener una imagen positiva frente a los sujetos de la muestra de ciencias; por otro lado, la radical diferencia entre la representación social desarrollada por los sujetos de la muestra de letras y ciencias nos lleva a pensar que puede haber existido un efecto de reactancia psicológica ante los intentos de influencia (Brehm; 1966, Brehm y Brehm, 1981). Varias razones apoyan esta hipótesis: En primer lugar, las recientes observaciones de Frey y Gneich (1984) demuestran que la gente siente una amenaza mayor en su libertad, ante los in- 
tentos de influencia, en privado que en público. En segundo lugar, mantener una posición favorable a los estudios de letras se opone, de alguna manera, a la tendencia general existente en la población. Es decir, el contenido mismo de las reivindicaciones es un buen portador de la intensidad particular del conflicto. Por último, el intento de influencia por parte de la minoría destacaría, en este contexto, la amenaza en su libertad para elegir en los sujetos de letras, máxime si se tiene en cuenta la concordancia entre las posiciones minoritarias y la identidad de los sujetos. La aparición de una reactancia psicológica se acompañaría de la reelaboración de la representación social de la minoría en términos negativos (Maass y Clark, 1986).

Es preciso señalar, asimismo, que el análisis factorial evidencia un cuarto eje referido al grado de autonomía de la fuente. Este factor resulta insensible a las manipulaciones experimentales. Los sujetos perciben el mismo grado de autonomía en todas las condiciones experimentales. Sin embargo, el que la variable independiente nivel de representatividad esté relacionada con la influencia obtenida plantea la necesidad de formular, en próximas investigaciones, un instrumento que permita dar cuenta de todas las dinámicas de la representación social en los procesos de influencia minoritaria. No obstante, creemos poder concluir, siguiendo las palabras de Papastamou, que «la influencia de una minoría dependerá esencialmente de la imagen que introduzca en la población». (1985, pág. 377).

\section{Referencias}

Aebischer V., Hewstone, M. y Henderson, M. (1984): «Minority influence and musical preference: Innovation by conversion not coercion». European Joumal of Social Psychology, $14,23-33$.

Brehm, J.W. (1966): A theory of Psychological Reactance. Nueva York, Academic Press.

Brenm, S.S. y Brenm, J.W. (1981): Psychological Reactance. A theory of Freedom and control. Nueva York, Academic Press.

Deschamps, J.C. (1977): L'attribution et la catégorisation sociale. Berna, Peter Lang.

Dosie, W. (1976): Larticulation psychosociologique et les relations entre groupes. Bruselas, De Boeck. En castellano (1977): Psicología Social y relaciones entre grupos. Barcelona, Rol.

DoIse, W. (1982): L'explication en psychologie sociale. París, Presses Universitaires de France.

FREY, D. y GNEICH, G. (1984): «Negative attitude change after a freedom-threatening influence», Manuscrito, Universidad de Kiel, F.R.G.

Hollander, E.P. (1960): «Competence and conformity in the acceptance of influence». Journal of Abnormal Social Psychology, 61, 361-365.

Lemaine, G. (1975): «Dissimilation and differential assimilation in social influence (situations of "normalization")». European Journal of Social Psychology, 5, 93-120.

Lemaine, G. (1984-85): «La différenciation sociale dans la communauté scientifique». Bulletin de Psychologie, 37, 477-488.

MaAss, A. y CLARK, R.D. (1984): "Hidden impact of minorities: fourteen years of minority influence research». Psychological Bulletin, 95, 428-450.

MAASS, A. y CIARK, R.D. (1986): «Conversion theory and simultaneous majority/minority influence: can reactance offer an alternative explanation?». Eumpean Joumal of Social Psycho$\log y, 16,305-309$.

MaAss, A., Clark, R.D. y Haberkorn, G. (1982): «The effect of differential ascribed category membership and norms on minority influence». European Journal of Social Pycbology, 12, 89-104.

Montmollin, G. (1977): L'influence sociale: phénomènes, facteurs et théories. París, Presses Universitaires de France.

MoscovicI, S. (1976): Social influence and social change. Londres, Academic Press. En castellano (1981): Psicología de las minorías activas. Madrid, Morata.

Moscovici, S., Lage, E. y Naffrechoux, N. (1969): «Influence of a consistent minority on the responses of a majority in a color perception». Sociometry 32, 365-380. 
Moscovici, S. y Mugny, G. (Eds.) (1967): Psychologie de la conversion: Etude sur l'influence inconsciente. Cousset, Delval.

Moscovici, S., Mugny, G. y Pérezz. J.A. (1984-85): «Effets pervers du déni (par la mayorité) de opinions d'une minorité». Bulletin de Psychologie, 38, 803-812.

Moscovici, S., Mugny, G. y Van Avermaet, E. (Eds.) (1985): Perspectives on minority influence. Cambridge y Parií, Cambridge University Press y Editions de la Maison des Sciences de l'Homme.

MugNy, G. (1975): «Negotiations, image of the other and the process of minority influence». European Joumal of Social Pychology, 5, 209-228.

MugnY, G. (1981): El poder de las minorías. Barcelona, Rol.

Mugny, G., Ibáñez, T., Elejabarrieta, F., Iñiguez, L. y Pérez.J. A. (1986): «Conflicto, identificación y poder en la influencia minoritaria». Revista de Psicología Social, 1, $39-56$.

Mugny, G., Kaiser, C. y Papastamou, S. (1983): «Influence minoritaire, identification et relations entre groupes: Etude expérimentale autour d'une votation». Cabiers de Psychologie Cognitive, 19, 1-30.

Mugny, G. y Papastamou, S. (1976-77): «Pour une nouvelle approche de l'influence minoritaire: Des determinants psychosociaux des strategies d'influence minoritaire». Bulletin de Psychologie, 30, 573-579.

Mugny, G. y PAPAstamou, S. (1982): «Minority influence and psychosocial identity». European Joumal of Social Pychology, 12, 379-394.

Mugny, G. y Papastamou, S. (1982-83): «Rigidité et influence minoritaires: le discours comme régulateur d'eppartenance». Bulletin de Psychologie, 36, 723-734.

Mugny, G. y Pérez,J.A. (1985): «Influence sociale, conflict et identification: Etude expérimental autour d'une persuasion "marquée" lors d'une votation». Cabiers de Psychologie Sociale, 26, 1-13.

Mugny,G. y Pérez,J.A. (1986): Le déni et la raison. Psychologie de l'impact social des minorités. Cousset, Delval.

Mugny, G. y Pérez, J.A. (1987): «Minorités, identification et influence». En S. Moscovicı y G. Mugny (Ed.): Psychologie de la conversion. Etude sur l'influence inconsciente. Cousset, Delval.

Mugny, G. y Pérez, J.A, Kaiser, C. y Papastamou, S. (1984): «Influence minoritaire et relations entre groupes: L'importance du contenu du message et des styles de comportement". Revue Suisse de Psychologie, 43, 331-351.

NemeTH, C. y WACHTLER, J. (1974): «Creating the perceptions of consistency and confidence: a necessary condition for minority influence». Sociometry, 37, 529-540.

Nemeth, C. y EnDICOTT, J. (1976): «The midpoint as an anchor: another look at discrepancy of position and attitude change». Sociometry, 39, 11-18.

Papastamou, S. (1985): «Effects de la Psychologization sur l'influence d'un groupe et d'un leader minoritaires». L'année psychologique, 85, 361-381.

Papastamou, S. (1986): «Psychologization and processes of minority and majority influence". European Joumal of Social Pychology, 16, 165.180.

Pérez,J.A. y Mugny, G. (1985a): «Categorización e influencia minoritaria». Anuario de Psicologia, 32, 97-116.

Pérez,J.A. y Mugny, G. (1985b): «Influencia minoritaria sobre las opiniones frente al aborto y los anticonceptivos". Estudios de Psicologia, 23/24, 29-53.

Pérez, J.A. y Mugny, G. (1986): «Efectos paradójicos de la categorización en la influencia minoritaria». Boletín de Psicología, 12, 65-89.

Pérez,J.A. y Mugny. G. (1987): «Paradoxical effects of categorization in minority influence: when being an outgroup is an advantage». European Joumal of Social Psychology, 17, 157-169.

Ricateau, Ph. (1970-71): «Processus de catégorisation d'autrui et les mecanismes d'influence sociale». Bulletin de Psychologie, 24, 909-919.

$T_{\mathrm{AJFEL}} \mathrm{H}$. (1978): Differentiation between social groups: studies in the social psychology of intergroup relations. Londres, Academic Press.

TAJFEL, H. (1981): Human groups and social categories. Cambridge, Cambridge University Press. En castellano (1984): Grupos sociales y categorías bumanas. Barcelona, Herder.

TAJFEL, H. y TURNER, J.C. (1979): "An integrative theory of intergroup conflict». En W.G. Austin y S. WORCHEL (Ed.): The social psychology of intergroup relations. Monterrey, Brooks Cole.

Turner,J.C. (1981): «Towards a cognitive redefinition of the social group». Cabiers de Psychologie Cognitive, 1, 93-118.

TURNER, J.C. (1984): «Social categorization and the self-concept: a social cognitive theory of group behaviourm. En E.J. LAw LeR (Ed.): Advances in Group Processes: Theory and Research, Greenwich: Connecticut, I.A.I. Press Inc.

ZaVALLONI, M. (1971): «Cognitive processes and social identity through focused introspection». European Journal of Social Psychology, 2, 235-260.

Zavalloni, M. y Luis-Guerin, C. (1984): Identité sociale et conscience. Introduction à l'ergoécologie, Privat, Les Presses de l'Université de Montréal, Privat. 